\title{
Outcome Predictors of Pediatric Extracorporeal Cardiopulmonary Resuscitation
}

\author{
Robert B. Kelly • Rick E. Harrison
}

Received: 14 August 2009/Accepted: 20 January 2010/Published online: 10 February 2010

(C) The Author(s) 2010. This article is published with open access at Springerlink.com

\begin{abstract}
Extracorporeal cardiopulmonary resuscitation (ECPR) allows clinicians to potentially rescue pediatric patients unresponsive to traditional cardiopulmonary resuscitation (CPR). Clinical and laboratory variables predictive of survival to hospital discharge are beginning to emerge. In this retrospective, historical cohort case series, clinical, and laboratory data from 31 pediatric patients $(<21$ years of age) receiving ECPR from March 2000 to April 2006 at our university-affiliated, tertiary-care children's hospital were statistically analyzed in an attempt to identify variables predictive of survival to hospital discharge. Seven patients survived to hospital discharge $(23 \%)$, and 24 patients died. Survival was independent of gender, age, and CPR duration. ECPR survival was, however, associated with a lower pre-ECPR phosphorus concentration $(P=0.002)$ and a lower pre-ECPR creatinine concentration $(P=0.05)$. A classification tree analysis, using, in part, a pre-ECPR phosphorus concentration threshold and a CPR ABG base excess concentration threshold, yielded a $96 \%$ nominal accuracy of predicting survival to hospital discharge or death. A large, multicenter, prospective cohort study aimed at validating these predictive variables is needed to guide appropriate ECPR patient selection. This study reveals the potential survival benefit of ECPR for pediatric patients, regardless of CPR duration prior to ECPR cannulation.
\end{abstract}

\footnotetext{
R. B. Kelly $(\varangle) \cdot$ R. E. Harrison

Department of Pediatrics, Division of Critical Care, David Geffen School of Medicine at UCLA, Mattel Children's Hospital UCLA, 10833 Le Conte Avenue, 12-494 MDCC, Los Angeles, CA 90095, USA

e-mail: rkelly@mednet.ucla.edu
}

Keywords Extracorporeal cardiopulmonary resuscitation - Cardiopulmonary resuscitation . Extracorporeal membrane oxygenation · Pediatric . Cardiothoracic surgery

Extracorporeal membrane oxygenation (ECMO) has been used in cases of pediatric cardiac arrest when traditional cardiopulmonary resuscitation (CPR) fails [2, 17, 20]. Survival using this modality has been demonstrated $[2,17$, 20]. This use of ECMO, also known as extracorporeal cardiopulmonary resuscitation (ECPR), allows clinicians to potentially rescue patients unresponsive to traditional CPR. In addition, not only does ECPR provide possibly desirable cardiopulmonary rest, but also ECPR may be used to bridge patients to subsequent orthotopic heart transplantation $[2,16,17]$ or a ventricular assist device [16].

The Extracorporeal Life Support Organization (ELSO) maintains an extensive registry of ECMO recipients. Collected data include demographic information, cannulation details, and ECMO complications $[10,11]$. According to the 2004 ELSO report, 151 neonatal cases and 282 pediatric cases of ECPR had been entered into the registry [7]. Among these cases, the neonatal ECPR survival rate to discharge was $43 \%$, and the pediatric ECPR survival rate to discharge was $39 \%$. In a recent review of pediatric ECPR, Thiagarajan et al. note that, between 1992 and 2005, there have been a total of 682 ECPR patients entered into the ELSO registry, with an overall survival rate of $38 \%$ [20].

Since ECPR typically requires significant medical and financial resources, the need for judicious patient selection is paramount. Similarly, since ECPR may cause significant morbidity, the ability to predict, prior to cannulation, which pediatric patients would benefit from ECPR and survive to 
hospital discharge would be advantageous. To our knowledge, this retrospective, historical cohort case series is one of the most inclusive and comprehensive analyses of multiple clinical and laboratory variables aimed at identifying appropriate pediatric candidates for ECPR. Although ELSO collects a significant amount of pediatric ECPR data, a number of critical data fields are not collected by the registry $[10,11]$.

The primary objective of this study is to identify clinical and laboratory variables predictive of survival to hospital discharge among pediatric patients suffering cardiac arrest and receiving subsequent ECPR. The secondary objective of this study is to analyze ECPR complications and outcomes. We hypothesized that the duration of CPR, performed by adequately trained health-care providers, has no effect on survival to hospital discharge in cases of pediatric ECPR. In addition, we hypothesized that the degree of acidosis prior to ECPR, as a measure of adequate ventilation and oxygen delivery, is inversely related to survival to hospital discharge.

\section{Materials and Methods}

This study was approved by the University of California, Los Angeles (UCLA), Institutional Review Board. The requirement for research informed consent was waived.

Mattel Children's Hospital UCLA is a university-affiliated, tertiary-care children's hospital within UCLA Medical Center. All pediatric ECMO patients are cared for in a neonatal, pediatric, or cardiothoracic intensive care unit. A dedicated pediatric ECMO rapid response team does not exist within UCLA Medical Center. Cardiothoracic surgery, pediatric surgery, and perfusion services, however, are readily available for consultation. ECMO cannulation is possible in multiple locations throughout the hospital.

All patients were identified from perfusion records maintained by Cardiac Perfusion Services at UCLA Medical Center. Medical records from all pediatric ECMO patients ( $<21$ years of age) between March 2000 and April 2006 were screened. Demographic, clinical, and laboratory data were collected and analyzed only from those patients receiving ECPR, as defined by ELSO [12]. According to ELSO, "ECPR denotes Extracorporeal Cardiopulmonary Resuscitation, in which ECLS [extracorporeal life support] was used as part of the initial resuscitation from cardiac arrest. Patients who are hemodynamically unstable and placed on ECLS emergently without a cardiac arrest are NOT considered ECPR." Cardiac arrest was defined as the need for chest compressions or direct, open-chest cardiac massage. Demographic, clinical, and laboratory data were recorded on a data abstraction tool approved by the UCLA Institutional Review Board.
Demographic data included each patient's age, gender, and underlying diagnosis. Laboratory data included the last serum sodium, potassium, chloride, bicarbonate, ionized calcium, magnesium, phosphorus, glucose, blood urea nitrogen, creatinine, and hemoglobin concentrations, as well as the last white blood cell and platelet counts, hematocrit, and arterial blood gas (ABG) results measured prior to, but not more than $24 \mathrm{~h}$ before, ECMO initiation. In addition, arterial blood gas data (worst values) obtained during CPR prior to ECMO initiation were collected. Clinical data included CPR duration, ECMO duration, ECMO complications, and survival to discharge. CPR duration was calculated from the time of initiation of chest compressions or direct, open-chest cardiac massage to the time of initiation of ECMO flow. ECMO duration was calculated from the time of initiation of ECMO flow to the time of ECMO flow discontinuation. Data from those patients for whom optimal ECMO flow could not be mechanically maintained were still included in the analysis if ECMO flow had been initially established.

The $P$ values for comparing continuous variables were computed using the nonparametric Wilcoxon rank-sum test. The $P$ value for comparing gender was computed using Fisher's exact test. A receiver operator characteristic (ROC)/discriminant analysis (nonparametric) was performed for each continuous variable to determine the best numerical threshold for predicting death (sensitivity) and survival to hospital discharge (specificity). The observed accuracy of predicting either death or survival to hospital discharge was calculated by averaging each variable's threshold sensitivity and specificity. Some continuous variables had skewed distributions on their original scale and were, therefore, examined on a logarithmic scale. For those variables with accuracies $\geq 80 \%, P$ values for comparing the proportions above the identified ROC/discriminant analysis thresholds were computed using Fisher's exact test.

Multivariate modeling utilized classification tree analysis, a specialized use of classification and regression tree analysis $[4,15]$. In our study, classification tree analysis, utilizing binary recursive partitioning, was used to divide study patients into either survivor or nonsurvivor groups by analyzing all variables (except gender and ECMO duration) and selecting those variables, along with their respective thresholds, which maximized the overall tree's accuracy in predicting either survival to hospital discharge or death. Patients with missing data are still included in classification tree analyses, but grouping proceeds after an analysis of alternate variables. Classification tree analysis allows for the identification of multiple variables and their respective thresholds, which, together, may improve the accuracy of predicting either death or survival to hospital discharge greater than any single variable alone. Each 
subsequent generation of nodes in a classification tree is the product of identifying the best variable and its respective threshold with the lowest $P$ value from among those subjects in the immediately preceding node. This process is repeated each time a new generation of nodes is produced, such that only the subjects comprising the immediately preceding node are analyzed, not the original group of subjects on which the first split was made in the classification tree. Therefore, although univariate or ROC/discriminant analyses may identify predictive variables one at a time while ignoring the other variables, classification trees identify predictive variables only from among those subjects comprising an individual node on which a new split is made. The classification tree, therefore, identifies predictive variables while controlling for (not ignoring) other variables previously identified.

Statistical analyses were performed utilizing SAS 9.1 (SAS Institute, Inc., Cary, NC) and Answer Tree 3.0 (SPSS Inc., Chicago, IL).

\section{Results}

Between March 2000 and April 2006, 31 pediatric patients at Mattel Children's Hospital UCLA received ECPR. The majority of ECPR recipients had a history of recent cardiothoracic surgery for a range of congenital cardiac defects (18 patients). Remaining patients had a history of remote cardiovascular surgery (six patients), cardiomyopathy (five patients), myocarditis (one patient), or pulmonary hypertension (one patient) (Table 1). Twenty-five patients suffered cardiac arrest in an intensive care unit, two patients in an operating room, one patient in the emergency department, one patient in a procedure room, one patient in a catheterization suite, and one patient on the inpatient ward. Of these 31 patients, 7 survived to hospital discharge (23\% survival), and 24 patients died. Survivor and nonsurvivor groups did not differ according to gender $(P=0.74)$ (Table 2).

Survivors to hospital discharge were statistically similar in age compared to nonsurvivors at the time of their cardiac arrest (median, 356 vs. 28 days; $P=0.07$ ). CPR duration

Table 1 Medical history of ECPR recipients $(N=31)$

\begin{tabular}{lll}
\hline Medical history & Survivors & Nonsurvivors \\
\hline Recent cardiothoracic surgery & 4 & 14 \\
Remote cardiothoracic surgery & 1 & 5 \\
Cardiomyopathy & 1 & 4 \\
Myocarditis & 1 & 0 \\
Pulmonary hypertension & 0 & 1
\end{tabular}

Note: ECPR, extracorporeal cardiopulmonary resuscitation
Table 2 Gender of ECPR recipients

\begin{tabular}{lll}
\hline Gender & Survivors & Nonsurvivors \\
\hline Male & 4 & 12 \\
Female & 3 & 12 \\
Total & 7 & 24 \\
\hline
\end{tabular}

Note: ECPR, extracorporeal cardiopulmonary resuscitation

did not differ between survivors and nonsurvivors (median, 40 vs. $47 \mathrm{~min} ; P=0.30$; range, $24-75$ vs. $5-101 \mathrm{~min}$ ). For two nonsurvivors, CPR initiation times were adjusted to a later time during the analysis due to an inability to precisely identify in the medical record the exact minute CPR was started. These two adjustments were judged to be minimal. Survivors and nonsurvivors were also found to have similar ECMO durations (median, 4 vs. 2 days; $P=0.15)$ (Table 3).

Survivors had significantly lower pre-ECMO serum phosphorus concentrations compared to nonsurvivors (median, 4.65 vs. $6.85 \mathrm{mg} / \mathrm{dl} ; P=0.002$ ). After log age adjustment, pre-ECMO serum phosphorus concentrations remained significantly lower among survivors (median, 4.96 vs. $6.70 \mathrm{mg} / \mathrm{dl} ; P=0.01)$. In addition, serum creatinine concentrations were lower among survivors (median, 0.6 vs. $0.8 \mathrm{mg} / \mathrm{dl} ; P=0.05$ ). Other laboratory variables did not appear to be associated with survival on bivariate analysis (Table 4).

Using ROC/discriminant analysis, pre-ECMO serum phosphorus concentration was found to be the most accurate variable predictive of survival to hospital discharge or death (threshold, $5.5 \mathrm{mg} / \mathrm{dl}$; sensitivity, $80 \%$; specificity, $100 \%$; accuracy, $90 \% ; P=0.001$ ), with a lower serum phosphorus concentration being associated with survival. Other highly accurate variables predictive of survival to hospital discharge included CPR ABG $p \mathrm{CO}_{2}$ concentration (threshold, $76 \mathrm{~mm} \mathrm{Hg}$; sensitivity, 67\%; specificity, 100\%; accuracy, $83 \% ; P=0.08$ ), with a lower CPR ABG $p \mathrm{CO}_{2}$ concentration being associated with survival, and CPR ABG bicarbonate concentration (threshold, $16.7 \mathrm{mmol} / \mathrm{l}$; sensitivity, $60 \%$; specificity, $100 \%$; accuracy, $80 \%$;

Table 3 Group comparison of clinical characteristics

\begin{tabular}{|c|c|c|c|c|c|c|c|}
\hline \multirow[t]{2}{*}{ Variable } & \multicolumn{3}{|c|}{ Survivors } & \multicolumn{3}{|c|}{ Nonsurvivors } & \multirow{2}{*}{$\begin{array}{l}P \\
\text { value }\end{array}$} \\
\hline & No. & Median & Mean & No. & Median & Mean & \\
\hline Age (days) & 7 & 356 & 1549 & 24 & 28 & 629 & 0.07 \\
\hline $\begin{array}{l}\text { CPR duration } \\
\text { (min) }\end{array}$ & 7 & 40 & 46 & 23 & 47 & 54 & 0.30 \\
\hline $\begin{array}{l}\text { ECMO duration } \\
\text { (days) }\end{array}$ & 7 & 4 & 4 & 24 & 2 & 6 & 0.15 \\
\hline
\end{tabular}

Note: $C P R$ cardiopulmonary resuscitation, ECMO extracorporeal membrane oxygenation 
Table 4 Group comparison of laboratory characteristics

\begin{tabular}{|c|c|c|c|c|c|c|c|}
\hline \multirow[t]{2}{*}{ Laboratory test } & \multicolumn{3}{|c|}{ Survivors } & \multicolumn{3}{|c|}{ Nonsurvivors } & \multirow[t]{2}{*}{$P$ value } \\
\hline & No. & Median & Mean & No. & Median & Mean & \\
\hline Sodium (mmol/l) & 7 & 143.0 & 141.1 & 24 & 145.0 & 145.7 & 0.32 \\
\hline Potassium (mmol/l) & 7 & 4.4 & 4.5 & 24 & 4.4 & 4.4 & 0.85 \\
\hline Chloride (mmol/l) & 7 & 104 & 104 & 24 & 106 & 106 & 0.21 \\
\hline Bicarbonate $(\mathrm{mmol} / \mathrm{l})$ & 7 & 22.0 & 23.0 & 24 & 21.0 & 22.3 & 0.45 \\
\hline Ionized calcium $(\mathrm{mmol} / \mathrm{l})$ & 6 & 1.16 & 1.25 & 22 & 1.33 & 1.36 & 0.26 \\
\hline Magnesium (mEq/l) & 7 & 2.0 & 2.0 & 22 & 1.9 & 1.9 & 0.63 \\
\hline Phosphorus (mg/dl) & 6 & 4.7 & 4.6 & 20 & 6.9 & 6.8 & 0.002 \\
\hline Glucose (mg/dl) & 5 & 230 & 206 & 23 & 174 & 197 & 0.72 \\
\hline Blood urea nitrogen $(\mathrm{mg} / \mathrm{dl})$ & 7 & 10 & 11 & 23 & 12 & 16 & 0.20 \\
\hline Creatinine (mg/dl) & 7 & 0.6 & 0.6 & 23 & 0.8 & 0.8 & 0.05 \\
\hline White blood cell count $(\times 1,000)$ & 7 & 11.3 & 10.6 & 20 & 12.4 & 12.8 & 0.41 \\
\hline Hemoglobin (g/dl) & 7 & 13.3 & 12.9 & 18 & 13.0 & 12.9 & 0.86 \\
\hline Hematocrit $(\%)$ & 7 & 39.4 & 38.8 & 22 & 36.3 & 37.7 & 0.68 \\
\hline Platelet count $(\times 1,000)$ & 7 & 168 & 166 & 20 & 123 & 145 & 0.32 \\
\hline Prothrombin time (s) & 6 & 14.6 & 14.7 & 16 & 16.8 & 24.7 & 0.21 \\
\hline Activated partial thromboplastin time (s) & 6 & 34.8 & 55.4 & 16 & 43.7 & 68.4 & 0.42 \\
\hline International normalized ratio & 6 & 1.4 & 1.5 & 16 & 1.6 & 2.1 & 0.32 \\
\hline Pre-CPR ABG pH & 4 & 7.33 & 7.31 & 22 & 7.20 & 7.21 & 0.36 \\
\hline Pre-CPR ABG $p \mathrm{CO}_{2}(\mathrm{~mm} \mathrm{Hg})$ & 4 & 44 & 47 & 22 & 49 & 58 & 0.75 \\
\hline Pre-CPR ABG $p \mathrm{O}_{2}(\mathrm{~mm} \mathrm{Hg})$ & 4 & 45 & 89 & 22 & 53 & 86 & 0.67 \\
\hline Pre-CPR ABG $\mathrm{HCO}_{3}{ }^{-}(\mathrm{mmol} / \mathrm{l})$ & 4 & 22.2 & 22.2 & 19 & 21.6 & 20.6 & 0.63 \\
\hline Pre-CPR ABG base excess (mmol/l) & 4 & -4.5 & -4.0 & 22 & -4.5 & -6.0 & 0.72 \\
\hline CPR ABG pH & 3 & 7.27 & 7.25 & 12 & 7.13 & 7.11 & 0.42 \\
\hline $\mathrm{CPR}$ ABG $p \mathrm{CO}_{2}(\mathrm{~mm} \mathrm{Hg})$ & 3 & 54 & 51 & 12 & 97 & 89 & 0.22 \\
\hline CPR ABG $p \mathrm{O}_{2}(\mathrm{~mm} \mathrm{Hg})$ & 3 & 43 & 103 & 12 & 35 & 89 & 0.31 \\
\hline $\mathrm{CPR} \mathrm{ABG} \mathrm{HCO}_{3}^{-}(\mathrm{mmol} / \mathrm{l})$ & 3 & 21.0 & 20.4 & 10 & 15.1 & 19.5 & 0.50 \\
\hline CPR ABG base excess (mmol/l) & 3 & -7.0 & -6.3 & 12 & -14.0 & -9.5 & 0.39 \\
\hline
\end{tabular}

Note. $\mathrm{CPR}$, cardiopulmonary resuscitation; $\mathrm{ABG}$, arterial blood gas

$P=0.19$ ), with a higher CPR ABG bicarbonate concentration being associated with survival.

A classification tree analysis, using, in part, a pre-ECPR phosphorus concentration threshold of $5.5 \mathrm{mg} / \mathrm{dl}$ and a CPR ABG base excess concentration threshold of $-13 \mathrm{mmol} / \mathrm{l}$, yielded a sensitivity of $92 \%$, a specificity of $100 \%$, and a nominal accuracy of predicting survival to hospital discharge or death of $96 \%$ (Fig. 1), with a lower pre-ECMO phosphorus concentration and a greater (integer value) CPR ABG base excess concentration associated with survival.

Upon reviewing ECPR complications and outcomes, following ECMO cannulation five patients $(16 \%)$ were found to have developed a new intracranial hemorrhage by radiographic or autopsy evidence. None of these patients survived to discharge. In addition, optimal ECMO flow could not be mechanically maintained for three patients $(10 \%)$. None of these patients survived to discharge. Three patients were ultimately declared brain dead. ECPR bridged one patient to orthotopic heart transplantation and ultimate survival to hospital discharge. In addition, ECPR successfully rescued one myocarditis patient who ultimately survived to hospital discharge.

\section{Discussion}

ECPR is a potentially lifesaving intervention, but one that carries significant risks. To our knowledge, this retrospective, historical cohort case series is one of the most inclusive and comprehensive analyses of multiple clinical and laboratory variables aimed at identifying appropriate pediatric candidates for ECPR.

Survival

Three recent reports have examined pediatric ECPR survival predictors $[2,13,20]$. Thiagarajan et al. have 


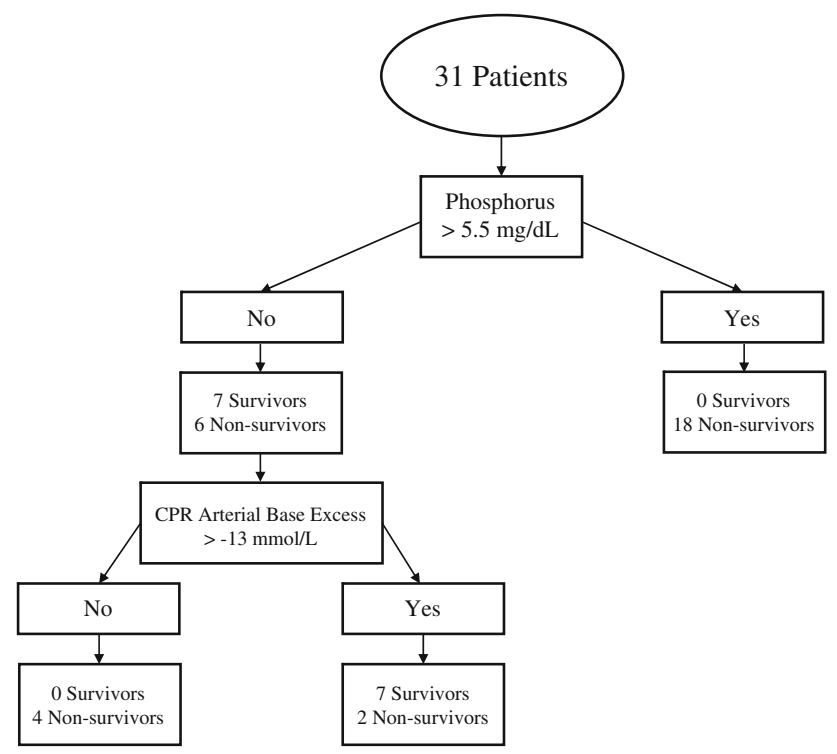

Fig. 1 Classification tree analysis of all variables (except gender and extracorporeal membrane oxygenation [ECMO] duration). Patients with missing data are included, but grouping proceeded after an analysis of alternate variables. The use, in part, of a pre-ECMO phosphorus concentration and a cardiopulmonary resuscitation (CPR) arterial base deficit concentration could predict survival to discharge or death with a nominal accuracy of $96 \%$

reviewed the largest series of pediatric ECPR cases known [20]. According to their report, the overall survival of all pediatric ECPR patients in the ELSO registry between 1992 and 2005 was 38\%. The survival rate among those patients receiving ECPR for cardiac reasons was $42 \%$. Although our survival rate is lower, our patients' illness severity and complexity of disease may not be comparable to those of patients in the ELSO registry. The majority of our patients had recent cardiothoracic surgery.

Although Alsoufi et al. have reviewed the second largest series of pediatric ECPR cases known, pre-ECMO laboratory variables were not examined [2]. Our data, however, are consistent with the finding that gender does not affect survival to hospital discharge. Furthermore, our survival rate to hospital discharge, although lower, is comparable (23 vs. 34\%). In another report, Huang and colleagues reviewed a series of 26 pediatric ECPR patients, including a number of clinical and laboratory variables [13]. A higher survival rate (41\%) was reported, despite our similar ECPR specialist availability and patient population.

The higher survival rate reported by Alsoufi et al. [2] is likely unrelated to the presence of an in-house ECMO specialist and/or perfusionist at all times at the Hospital for Sick Children in Toronto. Although UCLA Medical Center differs in that an ECMO perfusionist is not in-house at all times, our CPR duration times are very similar to those reported by Alsoufi and colleagues. In the report by Alsoufi et al., however, ECPR is considered within 10 min of starting
CPR. Likewise, our CPR durations were also similar to those reported by Huang and colleagues [13], but they had discussed the possible advantages of ECPR with staff, and the goal was to activate ECPR for appropriate patients after 10-15 min of refractory CPR. Although the survival benefit of a rapid response ECMO team does not appear statistically evident upon analysis of a report by Duncan et al. [9], this may be related to the small number of patients analyzed.

Mattel Children's Hospital UCLA does not have an official policy outlining the requirements needed to initiate ECPR. We speculate that educating staff and having a goal of activating or at least considering ECPR for appropriate patients early during CPR may introduce a selection bias. In other words, a physician may reject a potential ECPR candidate earlier due to a probable irreversible reason for the cardiac arrest. By then selecting a patient for ECPR because of a probable reversible reason for the cardiac arrest, the physician may be improving his or her institution's ECPR survival rate. We also speculate that educating staff and having a goal of activating or at least considering ECPR for appropriate patients early during CPR may improve or expedite the delivery of ancillary care, such as antibiotic or blood product administration. Although further investigation is needed to confirm these hypotheses, we speculate that being familiar with the potential advantages of ECPR and educating staff should, at least intuitively, improve ultimate survival to discharge.

\section{Phosphorus}

Pre-ECMO serum phosphorus concentration was found to be significantly lower among survivors to hospital discharge, even after log age adjustment. In addition, ROC/discriminant analysis identified the pre-ECMO serum phosphorus concentration as the most accurate continuous variable in predicting survival to hospital discharge or death. To our knowledge, these findings have not been reported previously. We further note that serum phosphorus concentrations among survivors to hospital discharge were not lower due to dialysis, as survivors to hospital discharge were not receiving dialysis at the time of their arrest.

We speculate that a serum phosphorus concentration in a compromised cardiac output state may be inversely related to a patient's metabolic reserve, as supported by $\mathrm{Ku}$ priyanov et al. in a porcine model of myocardial ischemia [14]. Increased phosphorus has also been observed in dogs (subjected to cardiac arrest) during CPR and following circulatory return [3]. Furthermore, increased phosphorus has also been seen after giving lactic acid to dogs [19]. The fact that specific CPR ABG $p \mathrm{CO}_{2}$ and bicarbonate concentration thresholds were also found to be highly accurate in predicting survival to hospital discharge or death by ROC/discriminant analysis lends support to this metabolic 
theory. Furthermore, a classification tree analysis, using, in part, a pre-ECPR phosphorus concentration threshold of $5.5 \mathrm{mg} / \mathrm{dl}$ and a CPR ABG base excess concentration threshold of $-13 \mathrm{mmol} / \mathrm{l}$, yielded a highly accurate model of predicting ECPR survival to hospital discharge or death, with a lower pre-ECMO phosphorus concentration and a higher CPR ABG base excess concentration associated with survival. Empirically, adequate ventilation, cardiac output, and end-organ perfusion, as reflected in normal CPR ABG $p \mathrm{CO}_{2}$, bicarbonate, and base excess concentrations, should be associated with patient survival. In other words, inadequate ventilation, cardiac output, and endorgan perfusion typically lead to acidemia from an increased $p \mathrm{CO}_{2}$ concentration and a decreased bicarbonate concentration as reflected in a decreased base excess concentration. Our findings regarding $\mathrm{CPR} A \mathrm{ABG} p \mathrm{CO}_{2}$, bicarbonate and base excess concentrations are consistent with this theory with regard to survival to discharge. This unexpected finding regarding phosphorus, however, requires validation.

\section{Creatinine}

Pre-ECMO creatinine concentration was found to be lower among survivors to hospital discharge $(P=0.05)$. One possible explanation for this finding is that a higher creatinine concentration represents pre-CPR compromised end-organ perfusion. Therefore, higher creatinine concentrations may reflect patients more susceptible to further impaired cardiac output during resuscitation.

Our finding conflicts, however, with other published reports and, therefore, requires validation. In a pediatric ECPR report by Huang and colleagues, the prevalence of "renal failure" (as defined by a low urine output or a fall in creatinine clearance) after ECMO initiation was significantly higher among nonsurvivors despite its having been similar prior to CPR. [13]. In another report, although Allan and colleagues found no difference in blood urea nitrogen or creatinine concentrations between survivors and nonsurvivors in their study of pediatric ECMO use associated with cardiac catheterization, 3 patients among their study population of 22 were not ECPR recipients [1].

\section{CPR Delivery}

ROC/discriminant analysis is a powerful statistical tool which identifies thresholds for continuous variables in cases of dichotomous outcomes, while maximizing sensitivity and specificity. This analysis may be particularly useful in clinical situations, where clinicians might be presented with a particular laboratory value and desire to predict the likelihood of one of two possible outcomes. ROC/discriminant analysis of our data, in addition to identifying a pre-ECMO phosphorus concentration threshold, also identified CPR ABG $p \mathrm{CO}_{2}$ and $\mathrm{CPR}$ ABG bicarbonate concentration thresholds as highly accurate predictors of survival to hospital discharge or death. Both CPR ABG $p \mathrm{CO}_{2}$ and CPR $\mathrm{ABG}$ bicarbonate concentrations likely reflect the adequacy of resuscitative ventilation, cardiac output, and end-organ perfusion and, thereby, the effectiveness of CPR in our patient population. This conclusion, however, is only speculative and requires further investigation. Similarly, the identification of a pre-ECMO phosphorus concentration threshold and a CPR arterial base excess concentration threshold in the multivariate analysis also supports our speculation that effective CPR is the major contributor to ECPR survival to discharge. For as discussed earlier, inadequate ventilation, cardiac output, and end-organ perfusion typically lead to acidemia from an increased $p \mathrm{CO}_{2}$ concentration and a decreased bicarbonate concentration as reflected in a decreased base excess concentration. Our findings regarding $\mathrm{CPR} A B G p \mathrm{CO}_{2}$, bicarbonate, and base excess concentrations are consistent with this theory with regard to survival to discharge. These laboratory tests may be surrogates for objective measurements of CPR performance, such as chest compression depth or ventilation frequency. Furthermore, by extension, those patients undergoing ineffective CPR may suffer proportionately more end-organ damage during CPR, thereby increasing their likelihood of mortality despite having survived cannulation. Interestingly, similar to our results, pre-ECPR acid-base status does appear to be associated with survival according to a report by Thiagarajan and colleagues [20]. ECPR survivors tended to have a lower pre-ECMO ABG $p \mathrm{CO}_{2}$ concentration $(P=0.06)$ and a significantly higher pre-ECMO ABG pH $(P=0.001)$.

\section{CPR Duration}

Whether CPR duration influences survival to discharge following ECPR is controversial. Two recent pediatric reports have shown that CPR duration is not significantly associated with death $[2,17]$. Our CPR duration times are comparable to these reports. Huang et al., however, showed a significant association between longer CPR duration and death among pediatric ECPR recipients [13]. In their report, ECPR "activation time" ("time from cardiac arrest to calling the ECMO team") took significantly longer for those patients who ultimately did not survive. In addition, a large adult ECPR study has indicated that a CPR duration $<60 \mathrm{~min}$ is significantly associated with survival [6]. Our series would suggest that CPR duration does not affect survival to discharge among pediatric patients receiving ECPR. We speculate that this may be due to the fact that effective CPR depends, in part, on qualified providers, adequate vascular access, ready availability of resuscitation 
medications and equipment, and optimal hemodynamic monitoring. These are typically present in tertiary-care settings, such as ours. As a result, we speculate that CPR duration is likely less important to ECPR survival to discharge than the effectiveness of that CPR, perhaps reflected by, as we speculate, CPR ABG $p \mathrm{CO}_{2}$, bicarbonate, and base excess concentrations, for example. This is only a hypothesis, however, requiring further investigation.

\section{ECMO Duration}

ECMO duration did not differ significantly between survivors to hospital discharge and nonsurvivors. This finding is consistent with other reports [2, 5, 13, 17]. At our institution, although the timing of ECMO decannulation procedures varied widely and an intracranial hemorrhage is generally a contraindication to ECMO continuation, this finding may indicate that providing adequate pre-ECMO oxygen delivery affects overall survival to hospital discharge more than the ECMO course, itself, and any ECMO complications.

\section{ROC/Discriminant Analysis}

As noted previously, ROC/discriminant analysis is a powerful statistical tool which identifies thresholds for continuous variables in cases of dichotomous outcomes, while maximizing sensitivity and specificity and, thereby, accuracy. This analysis is provided as an alternative and, perhaps, more clinically relevant way to analyze our ECPR data as opposed to traditional univariate distribution comparisons. $P$ values computed for continuous univariate and ROC/discriminant analyses, respectively, may not be equivalent since ROC/discriminant analysis, unlike traditional univariate analysis, identifies an actual threshold that maximizes accuracy. ROC/discriminant analysis, therefore, may be particularly useful in clinical situations where clinicians are presented with a particular laboratory value and desire to predict the likelihood of one of two possible outcomes. ROC/discriminant analysis can, therefore, realistically guide clinical decisions at the bedside during ECPR candidate evaluation, especially if the threshold for the variable in question has a high accuracy. ROC/discriminant analysis can potentially provide useful information that can guide clinical decisions when variables with high accuracy have been identified. Our findings, however, first require validation.

\section{Limitations}

Our retrospective, historical cohort case series has a number of limitations potentially affecting the ability to generalize our findings to other institutions, providers, and patients. Our study population, although largely homogeneous, was relatively small. A larger sample size may be required to see larger or additional differences between survivors to hospital discharge and nonsurvivors. Also, all of our patients suffered in-hospital cardiac arrests, which are associated with improved survival to discharge, compared to out-of-hospital arrests $[8,18,21]$. Furthermore, multiple medical providers were involved in the care of these patients, and variations in medical and surgical care undoubtedly occurred, before, during, and after cannulation. We also did not analyze any potentially available follow-up records, including neurological and developmental evaluations. Finally, the number of physicians who rejected potential ECPR candidates due to probable irreversible reasons for their cardiac arrest (selection bias) is unknown.

\section{Conclusions}

ECPR is a potentially lifesaving intervention, but one that carries significant risks. Lower pre-ECMO phosphorus and creatinine concentrations were associated with survival to hospital discharge in our patients. Furthermore, CPR arterial $p \mathrm{CO}_{2}$, bicarbonate, and base excess concentration thresholds may aid in the prediction of patient survival to discharge or death.

We believe that a large, multicenter, prospective cohort study is required to sufficiently validate whether these preECPR variables or others are predictive of survival to hospital discharge. Selection bias, however, may be difficult to eliminate. Such a study could potentially identify differences among certain subgroups of patients with specific diagnoses. Additional data fields should be collected as well.

We strongly caution against generalizing these findings to other patients. Validation of these results and further investigation are needed. At the very least, this study provides statistical methods and a framework for analyzing this clinical dilemma. Given the interplay of many variables and the complexity of human physiology, a multivariate model is likely to provide the most guidance. Fortunately, the laboratory variables found to be associated with survival to hospital discharge in this report are readily available in the modern medical setting. Given the limitations discussed, however, pediatric cardiologists, intensivists, and surgeons should continue to rely on clinical judgment when evaluating potential ECPR recipients. As our data support, ECPR should remain an option to healthcare providers in cases of refractory pediatric cardiac arrest, even if CPR duration is lengthy.

Acknowledgments Funding for this research was provided by the Division of Critical Care, Department of Pediatrics, Mattel Children's Hospital UCLA. We thank Dr. Jeffrey Gornbein and Ms. Daniela 
Markovic, Statistical Biomathematical Consulting Clinic, UCLA Department of Biomathematics, for providing the statistical analyses. We also thank Dr. Anthony C. Chang, Children's Hospital of Orange County Heart Institute, for his thoughtful review of the manuscript.

Open Access This article is distributed under the terms of the Creative Commons Attribution Noncommercial License which permits any noncommercial use, distribution, and reproduction in any medium, provided the original author(s) and source are credited.

\section{References}

1. Allan CK, Thiagarajan RR, Armsby LR, del Nido PJ, Laussen PC (2006) Emergent use of extracorporeal membrane oxygenation during pediatric cardiac catheterization. Pediatr Crit Care Med 7:212-219

2. Alsoufi B, Al-Radi OO, Nazer RI, Gruenwald C, Foreman C, Williams WG, Coles JG, Caldarone CA, Bohn DG, Van Arsdell GS (2007) Survival outcomes after rescue extracorporeal cardiopulmonary resuscitation in pediatric patients with refractory cardiac arrest. J Thorac Cardiovasc Surg 134:952-959

3. Bleske BE, Song J, Chow MS, Kluger J, White CM (2001) Hematologic and chemical changes observed during and after cardiac arrest in a canine model - a pilot study. Pharmacotherapy 21:1187-1191

4. Breiman L, Friedman JH, Olshen RA, Stone CJ (1984) Classification and regression trees. Wadsworth International Group, Belmont, CA, pp 18-58

5. Cenqiz P, Seidel K, Rycus PT, Brogan TV, Roberts JS (2005) Central nervous system complications during pediatric extracorporeal life support: incidence and risk factors. Crit Care Med 33:2854-2855

6. Chen YS, Chao A, Yu HY, Ko WJ, Wu IH, Chen RJ, Huang SC, Lin FY, Wang SS (2003) Analysis and results of prolonged resuscitation in cardiac patients rescued by extracorporeal membrane oxygenation. J Am Coll Cardiol 41:197-203

7. Conrad SA, Rycus PT, Dalton H (2005) Extracorporeal life support registry report 2004. ASAIO J 51:4-10

8. Donoghue AJ, Nadkami V, Berg RA, Osmond MH, Wells G, Nesbitt L, Stiell IG, for the CanAm Pediatric Cardiac Arrest Investigators (2005) Out-of-hospital pediatric cardiac arrest: an epidemiologic review and assessment of current knowledge. Ann Emerg Med 46:512-522

9. Duncan BW, Ibrahim AE, Hraska V, del Nido PJ, Laussen PC, Wessel DL, Mayer JE Jr, Bower LK, Jonas RA (1998) Use of rapid-deployment extracorporeal membrane oxygenation for the resuscitation of pediatric patients with heart disease after cardiac arrest. J Thorac Cardiovasc Surg 116:305-311

10. Extracorporeal Life Support Organization. ECLS cardiac addendum form. Available at: http://www.elso.med.umich.edu/ WordForms/ELSOCardiacForm.pdf. Accessed April 25, 2008

11. Extracorporeal Life Support Organization. ECLS registry form. Available at: http://www.elso.med.umich.edu/WordForms/ELSO RegistryForm.pdf. Accessed April 25, 2008

12. Extracorporeal Life Support Organization. Registry form instruction sheet. Available at: http://www.elso.med.umich.edu/ WordForms/FormInstruction.doc. Accessed April 25, 2008

13. Huang SC, Wu ET, Chen YS, Chang CI, Chiu IS, Wang SS, Lin FY, Ko WJ (2008) Extracorporeal membrane oxygenation rescue for cardiopulmonary resuscitation in pediatric patients. Crit Care Med 36:1607-1613

14. Kupriyanov VV, Dai G, Shaw RA, Sun J, Jilkina O, Luo Z, Mantsch H, Deslauriers R (2000) Noninvasive assessment of cardiac ischemic injury using ${ }^{87} \mathrm{Rb}$ and ${ }^{23} \mathrm{Na}$ MR imaging, ${ }^{31} \mathrm{P}$ MR, and optical spectroscopy. Magn Reson Med 44:899-908

15. Lewis RJ (2008) An introduction to classification and regression tree (CART) analysis. Available at: http://www.saem.org/ download/lewis1.pdf. Accessed April 27, 2008

16. Massetti M, Tasle M, Le Page O, Deredec R, Babatasi G, Buklas D, Thuaudet S, Charbonneau P, Hamon M, Grollier G, Gerard JL, Khayat A (2005) Back from irreversibility: extracorporeal life support for prolonged cardiac arrest. Ann Thorac Surg 79:178183

17. Morris MC, Wernovsky G, Nadkarni VM (2004) Survival outcomes after extracorporeal cardiopulmonary resuscitation instituted during active chest compressions following refractory inhospital pediatric cardiac arrest. Pediatr Crit Care Med 5:440-446

18. Nadkami VM, Larkin GL, Peberdy MA, Carey SM, Kaye W, Mancini ME, Nichol G, Lane-Truitt T, Potts J, Ornato JP, Berg RA, for the National Registry of Cardiopulmonary Resuscitation Investigators (2006) First documented rhythm and clinical outcome from in-hospital cardiac arrest among children and adults. JAMA 295:50-57

19. Oster JR, Alpert HC, Vaamonde CA (1984) Effect of acid-base status on plasma phosphorus response to lactate. Can J Physiol Pharmacol 62:939-942

20. Thiagarajan RR, Laussen PC, Rycus PT, Bartlett RH, Bratton SL (2007) Extracorporeal membrane oxygenation to aid cardiopulmonary resuscitation in infants and children. Circulation 116: 1693-1700

21. Young KD, Gausche-Hill M, McClung CD, Lewis RJ (2004) A prospective, population-based study of the epidemiology and outcome of out-of-hospital pediatric cardiopulmonary arrest. Pediatrics 114:157-164 